therefore a far greater uniformity is obtained in the product from that card.
As the previously mentioned lattice or apron which receives the lap operates at right angles to the film the filament must enter the feed rolls of the following card at right angles, or sidewise instead of end wise. of disentanglement and rearranging while passing the same system is employed for conveying the woo from the second breaker to the finisher, a third disen tinual working of the delicate fibers is not recommendnecessitating an excess of strength, elasticity and duracarding must neecessarily produce a comparatively and similar defects in the staple, than the method of presenting the fibers to the following machines in subof the preceding one.-Manufacturers'

OIL AND IRON STAINS IN COTTON CLOTH OIL stains in cotton cloths are an occurrence wel
known to every bleacher and dyer, and it is the general experience that their completo removal is effected where the oil stains have been caused by animal or vegetable oils and greases, as in this case, under the
circumstances obtaining in keirs, the saponification of these oils completely removes the stains. Not quite oils. These are incapable of saponification, but as soap solutions (especially alkaline ones) dissolve consumed that the resin soap employed in the process of stains. This may be true as long as the stains are long exposure to the air have undergone oxidation. fectively dealt with in an open keir, although in a pres-
sure keir, and conditional to a liberal supply of resin soap, the stains practically disappear, $i$. $e$., they can
no longer be seen; and in the process of printing or dyeing such cloth, nothing occurs that would indicate perhaps, occur not so often in cloth as oil stains, and ordinary circumstances their removal is easy enough. one by one with a moderately strong solution of oxalic
acid, the piece being subsequently washed. If there treatment, padding in a bath of oxalic acid at 5 . Tw. or in bisulphite of soda at $7^{\circ}$ Tw. will answer. 5 . If, however, oil and iron stains appear in the same
piece, forming as it were one single stain, the question of getting rid of these combined stains is in most
cases a matter of very considerable difficulty, the oxicases a matter of very considerable difficulty, the oxiacid ; even the most powerful agent for removing iron stains, a solution of tin oxalates in hydrochloric acid, has not the slightest effect on these compound stains. stains ever being found in gray cloth, or being prothe single oil or iron stains are common enough at this stage. But the compound stain inevitably forms when
oil-stained cloth is dyed with an iron mordant. The oil-stained cloth is dyed with an iron mordant. The found out by treating a suspected sample in a bath of

soda carbonate.

As a rule the stain does not show in the buff, rout
after stripping the color in any suitable acid bath. a bright iron stain remains wherever the cloth retained the least trace of an oil stain. From this it is clear
that in the majority of cases these compound stains will never be noticed, unless the cloth is stripped of
its dye. Unfortunately the latter process is frequently its dye. Unfortunately the latter process is frequently
necessary in the case of drab twills, which have at
times, from some unaccountable reason, tendency to bleach in the folds, or to come up a wrong tendency to bleach in the folds, or to come up a wrong
pieces the color is stripped and then the oil stains become visible as bright iron stains. On redyeing these
pieces in the manner generally used for this class of pieces in the manner generally used for this class of
goods, by first giving two ends in a mixed bath of fustic, sumac, and annatto, and afterward fixing in a bath of ferrous sulphate, these iron stains do not disappear,
but show as ugly olive patches. That these stains show only in the second dyeing is easily accounted for, as they now contain twice as much iron as the rest of pieces stained in this manner, it is absolutely necessary
to first remove these stains. I have already mentioned
the obstinacy with which these stains resist all ordinary agents, and the cause of this, no doubt, is that we have the iron here in the form of an iron soap. Taking stain will only yield if treated with an agent which at
the same time loosens the oil stain. After a great many experiments I found that by padding such preces in a hot solution of one part of soft soap, one part of gly-
cerine, and three parts of water, taking through squeezing rollers, letting lie for 24 hours, then washing, the
iron stains, together with the oil stains, are completely removed. The rationale of the process will be readily understood if we remember the great ease with which
oils of every description dissolve in solutions of glycerine and soap, and also the capability of alkaline glyties. The price of the process amounts to about 3s. per may easily be calculated, the weight of a piece varying from 26 to about $80 \mathrm{lb}$

The whole difficulty about these compound stain would of course best be dealt with by taking care to
remove every trace of oil in the cloth in the keiring process, but as I have already remarked, this is a mat- Tobacco is a poison as are most of the Solanace stains, althouble difficulty in the case of mineral oil $\mid$ and many plants which medicine daily utilizes. It stains, although pressure keirs are, as a rule, fairly properties have been studied in our time with all the of an oir is then quite sufficient to remove every trace
Industry.

TOBACCO AND THE TOBACCO HABIT. By M. Jules Rochard, of the French Academy THE use of tobacco prevails throughout the whole
orld. Smokers alone are numbered by hundreds million franes (or sixty million dollars). A custom so
general, a habit that has been maintained so long in the face of constant attacks upon it, should be
sidered seriously. It should be studied from ondness for their habit, while those who have been proselytes; but I intend to say what I believe is true
upon a question which I have studied well, and on terminating in uneven teeth. The genus Nicotiana
neludes some fifty other species, mostly natives of
America, but some of Australia and the islands of the America, but some of Australia and the islands of the A few species, remarkable for the richness of their
colors and their graceful growth, are cultivated as Tobacco leaves contain princ

regetable substances-such as starch, cellulos to a rganic acids, and salts-principles soluble in ether
itrogenous substances, and a peculiar alkaloid to nitrogenous substances, and a peculiar alkaloid to
which the plant owes its special qualities, called nico-
tine. This alkaloid, discovered by Posselt and Remann, was isolated by Vauquelin in 1809 . It is an oily and thick in the air by absorbing oxygen. Its acrid and virulent odor is like that of tobacco; it has
burning taste, and its vapor is so irritating that breath ing is painful in a room where a drop of it has fallen.
It is very hygrometric, and soluble in water, aleohol,
and ether. It combines directly with acids, with the quantity of it increases with the development of the
plant, and varies according to the thickness of the
leaves. The thinner-leaved plants contain less of it. The fermentation to which tobacco is subjected in The fermentation to which tobaceo is subjected in substitutes ammonia for it. Consequently, there is less
nicotine in tobacco prepared for consumption than there was in the dry leaves before the preparation.
Combustion destroys about three-quarters of this. According to M. Pabst, the smoke of five grammes of
tobacco yields about three milligrammes of nicotine; but it contains a number of other principles besides, the enumeration of which here would not be interest-
ing. Nicotine is the active principle of tobacco, as there are other poisons among the substances united bustion, and produce a brownish empyreumatic liquid,
kind of coal tar of tobacco a part of which a kind of coal tar of tobacco, a part of which oozes
through porous pipes, and the whole of which is retained in the water of nargilehs.
Among the volatile principles that pass into the smoke along with nicotine are hydrocyanic acid and lowing the smoke, and the gas passes into the stream importance in view of practical consequences, and go far to explain the accidents that sometimes occur afte ith tobaceo, even without smoking, and the phenom na of intoxication which are produced by eating out on a large scale, it appears, however, that this
difficulty can be overcome by deliberately increas-
cipal results of the investigation here. The decoction difficulty can be overcome by deliberately increas- cipal results of the investigation here. The decoction on a vegetable oil to it. Treatment even in an portion to the strength of the dose. The phenomena millions. A million and a quarter acres of the earth
are devoted to the cultivation of the plant, and the are devoted to the cultivation of the plant, and the
million on it alone in France amount to three hundred
mixty million dollars). A custom so side, and the various elements of the question should
be subjected to a complete analysis by the means of investigation now at our disposal, for it is a scientific
problem of the first order. While it is of moral and
philosophical interest, and its social consequences are within the provinee of economists, it is for science,
physiology, and hygiene to furnish experimental data as the basis for their deductions.
A proper study of the subject should be made with an independence of prepossession which is not easy to
find. Persons who have never smoked will talk of
tobacco as the blind talk of colors : smokers have a obliged to give it up are prejudiced on the other side.
I am one of the reformed smokers. After having
abused tobacco for about fifty years. I was compelled abused tobaceo for about fifty years, I was compelled yielded only to an absolute necessity. Knowing what upon a question which I have studied well, and on
which I am not lacking in personal experience.
The tobaceo plant belongs to the order solanacea, and constitutes a genus (Nicotiana) named after Jean
Nicot. It is cultivated through the whole world, and succeeds equally in the temperate zone and the inter-
tropical regions. Two species are cultivated : common or large tobacco (Nicotiana tabacum) and small
tobacco (Nicotiana rustica). The first species is the most widely diffused. It is a large and fine looking
annual plant, growing to a height of about six feet. It bears large alternate leaves of a glaucous green having a pale rose corolla and a persistent five-parted green, and viscously hairy. The terminal inflorescence
comprises clusters of flowers composed of cymes. The pale yellow corolla, a little creetish, is supported by a
campanulate calyx, eovered with glandular hairs and Pacitie Ocean. Of these, some fifteen or twenty evolution of heat. It is found as a malate in the
leaves. The different kinds of tobacco do not contain
the same quantities of it. The black, unctuous tobacco the same quantities of it. The black, unctuous tobacco
of the Antilles, the pronounced savor, ready burning, of the Antilles, the pronounced savor, ready burning,
and white ash of which make it in demand among
experienced smokers, contains much more nicotine xperienced smokers, contains much more nicotine
than the light, fragrant tobacco of the Levant. The carbonic oxide. Dr. Grehant has shown that a notable
quantity of them is absorbed by rapid smokers swal-
lowing the smoke, and the gas passes into the stream portion to the strength of the dose. The phenonten
alkaloids death are like those produced by other toxic
man in a similar condition, whith those exhibited by doctors have had man in a similar condition, which doctors have had
too frequent occasion to observe. Sometimes convicts wager a glass or two of the empyreumatic juice that
flows from old pipes, or the poison is swallowed by mistake, as when snuff is taken for coffee or tobaceo
leaves are mixed with orange leaves. Cases of mali-
cious poisoning are more rare; but the poet Sauteuil cious poisoning are more rare; but the poet Sauteuil having drunk a glass of wine in which Spanish tobaceo rarely brought about when tobaceo is taken by the
mouth, for it is nearly always rejected by vomiting before it can produce its worst effects : but the results
of intestinal administration are different. The intoxication is then most usually the result of a medical
error. The decoction of tobacco is still given sometimes as an injection of tobacco is still given somesion or of strangled hernia, and, if the dose is too large fatal in doses ranging from eight to sixty-four grammes. One patient died in fifteen minutes, and the one who grammes do not form a toxic dose, but the case cited
by Orfila was one of an infant. From fifteen to thirty grammes are required to kill an adult. Tobacco may
also poison through the lungs. Cases are mentioned of persons who died from sleeping in a room filled with ermenting leaves; others, worthy rivals of the bettors
ust now spoken of, died after executing wagers that they could smoke an improbable number of pipes
without intermission. The skin itself may serve as a channel for the introduction of the toxic principle.
Accidents of this kind were not rare when diseases of the skin were treated with pomades or liniments of which tobaceo was the base. Murray reports an
observation of three infants who were taken with vomitings and vertigos, and died in convulsions within
twenty-four hours after having their heads rubbed with a four hours after having their heads rubbed smugglers who died after having covered the bare skin
of their bodies with tobacco leaves which they were trying to introduce fraudulently. Ferdinand Martin
has related the case of a lady afficted with lumbago who applied flannels dipped in a decoction of smoking subdued, but she soon felt all the phemonena of intoxication by nicotine, and did not recover from it for
three days. Poisoning by tobacco generally oceurs by
accident or mistake. It is rarely tried criminally, probably because the toxic properties of the drug are not reliable enough. Assassins prefer the alkaloid
itself, the effects of which are much more prompt and more terrible than those of the plant. By whatever is slain. Two drops are enough to kill a large dog;
eight drops will kill a horse in four minutes. Under ts effects he rages, prances, writhes, falls down, and
dies in convulsions. "This alkaloid," says Claude dies in convulsions. "This alkaloid," says Claude
Bernard,* "is one of the most virulent poisons known, and a few drops of it on the cornea of an animal will its effects, in its action is very much like prussic acid." be analyzed unless the drug is administered in minute doses and very dilute solutions.
served the phenomenon-which goes far to explain
the fath which one is habituated to the use of tobacco-of the rapid development of toleranee of gradually increasing doses. This has been demonstrated by Traube, who, with the twenty-fourth of a
drop of nicotine subcutaneously injected, obtained very the same animal, it took a whole drop to reach the
same result, and at the end of four days five drops were
necessary. A similar tolerance is observed in man for hypodermic injections of morphine; but one does not When nicotine is administered in doses weak enough
Whita phenomena are witnessed as with the whole. plant. In the cases of poisoning already mentioned, there came the cases of poisoning already mentioned, there came with sensations of burning heat in the pit of the stomslackened; then came vomiting and purging, vertigo,
and faintness. The face grew pale, the skin was and faintness. The face grew pale, the skin was
covered with a cold sweat, the head was confused, and the patient fell into a deep stupor, with cories, general trembling, and convulsions. This agitation gives place
to paralysis and insensibility ; respiration is impeded,
the pulse declines to a mere thread, and the patient dies in syncope.
When the patient resists the attack, as is most frequently the case, the evolution of the symptoms
described above is arrested, and the sufferer comes out of his comatose condition with a violent headache, equires a considerable time to allay.

The eff ects produced by the habitual use of tobacco differ according to the way it is consumed. They have not been much observed except among smokers, who
are most noticed because of their number. Then their causes inconvenience to others : while the more discreet snuff taker can hide his snuff box, and annoys
with the smell of tobaceo only those who come too near him. Beginners at snuff taking require, like smokers, an
apprenticeship. They begin by sneezing; then the mucous membrane of the nasal fossa becomes, accuspleasantly tickled by the ammoniacal piquancy and becomes thick. and with intemperate snuff takers perceives odors only feebly. It becomes sometimes the seat of a chronic inflammation which extends to the
pharynx and produces a slight dry and characteristic from eruptions, ulcerations, and polypi ; others have 
become deaf ; but such vases are so rare and their life in them upon the operatives has been painted in $\mid$ day do not have to make the same struggles, but many etiology is so doubtful that serious account need not very dark colors. All manufactories were pntil re- of them endure troubles that compromise their health
be taken of them. The of them. observed among snuff takers is a rhythmic trembling ards, but which is observed likewise in excessive smokers. A single case is mentioned by Dr. Bean of excessive use of snuff. But a solitary case is not im portant in the consideration of a habit so general, and taking is condemned by fashion, from whose decree there is no appeal. Therative.
imperat so Smoking is charged by its opponents with injuring
the health and debasing the mind. The former part of the charge has a measure of foundation. There is cerquainted with the troubles that ensue on the first
effort to smoke. There are nauseas, soon followed by vomiting, headache, vertigo, and a condition resembling sea sickness, and much like the earlier phenomena
of acute poisoning by tobacco. These troubles soon
pass a a way, and after a few succeeding pass away, and after a few succeeding efforts the When the habit is once acquired, smokers feel no
further inconvenience; and there are some who are further inconvenience; and there are some who are
able to smoke just before sitting down at the table.
Smoking generally dulls the appetite and gives relief against the pains of hunger. But after eating the against to smoke becomes irresistible. This is the
psychological moment; and the pleasure we feel then psychological moment; and the pleasure we feel then The pipe or the cigar is a condition of good digestion troubles. Nervous people, those who lead a too
sedentary life, and office men, especially if they have the habit of smoking before meals, gradually lose their
appetite, and acquire instead of it a painful anxiety
and nausea. Others suffer from pyrosis. There are and nausea. Others suffer from pyrosis. There are day without having the feeling of hot iron that marks peptics: and the fact is explained by the excess of salivation and the diminution of the gastric juice and the dipestive troubles, the most common affections pharyngitis is very common among persons who
smoke to excess. The irritation of the pharynx is often communicated to the larynx, and there results a
peculiar dry cough. Others feel a temporary oppression in the evening after having smoked during the caused by the abuse of tobacco; but cases of it must
be very rare, for I have never observed it, though I have
passed my life among smokers. Affections of the heart passed my life among smokers. Affections of the heart consequent to their indolence and a relief from its are more frequent. Some doctors assert that one- been pushed further, it would have been found that
fourth of the smokers are afficted with palpitations the same and irregularities of the pulse. I do not know where most to be desired, and who are most frequently

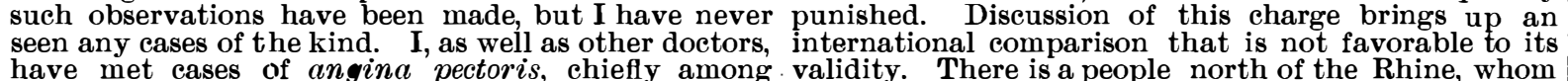
have met cases of angina pectoris, chiefly among . validity. There is a people north of the Rhine, whom rated with tobacco, and among those who have swal- tobacco has almost become an institution. They conlowed the smoke of their cigars, and have not been surprised at them, because the smoke then enters into the lesser ramifications of the bronchial vessels, where
lungs aud the heart, and its action induces the of thasms
of suffocation that constitute that terrible disease.
These symptoms are at first fleeting, and rarely mortal; These symptoms are at first fleeting, and rarely mortal;
but, if the patient does not abandon his habit, they
occur more frequently, and become more grave till occur more frequently, and become more grave till
death ensues in one of them. Disasters from breathing an atmosphere saturated with tobacco smoke seem
more liable to occur with children than with grown persons. Staying in smoking rooms, where the smoke end of the room to the other, is dangerous to persons
subject to palpitations, even though they do not to this point, one of which relates to the case of a young officer who had given up tobacco three months culprit is a reason why it should not be painted blacker angina pectoris after having passed several nights in imaginary ones to it, we run the risk of wholly missing his room, where his friends canie to smoke for some our aim. In fact, children whom we are trying to pre-.
hours every evening. Dr. Gelineau tells of an epi- serve from it, when they see smokers around them demic of an gina pectoris among some sailors who able bodied and sparkling with wit, are disposed to were crowded in the between decks of a merchant think we are deceiving them when we hold up this
vessel during a storm that made it necessary to close bugbear before them, and wili come to not believing
all the hatches, and who smoked to pass away the the real evils of the bad habit against which we are tinie. Those who did not join in the smoking suffered equally with the others, for they breathed the same question : What is the motive that impels so many
Pipe smokers are in danger of epithelioma, or cancer persons to contract an inconvenient, expersive, and
of the lips and of the tongue. The former occurs unhealthy habit? The problem is insoluble to persons "
chiefly among persons who smoke a very short-stemmed who do not smoke. "I can never comprehend" lately Last to be considered is the philosophical side of the
Lang the chiefly among persons who smoke a very short-stemmed who do not smoke. "I can never comprehend,"lately
clay pipe. Smoker's cancer appears usually at the said a professor of hygiene, "the enjoyment one can
point where the hot pipe stem bears upon the lower lip, feel in converting his mouth into a chimney flue" point wh the side of the tongue at the point where the Dupuytren called the habit of smoking the ignoble smoke touches at each aspiration. Ir1 some cases it pleasure of poisoning one's self and others. This is not begins with buccal psoriasis, a kind of thickening of surprising; but it is more so that smokers themselves
the epithelium of the tongue, which kecomes white, cannot account for the fact. The general opinion is
glossy, and horny. These two forms of a horrible that we begin to smoke to imitate others, and continue glossy, and horny. These two forms of a horrible that we begin to smoke to imitate others, and continue malady are incontestably the most serious danger it by habit, as a distraction, or means of dispelling
smokers incur : and the fear of it is the motive that ennui. "The boy of fourteen or fifteen years, begin-
has impelled the majority of conversions from the ning to smoke," says M. Dumas, "does not seek a
habit. The frequency of them should not, however, cerebral excitement in the new habit any more than habit. The frequency of them should not, however,
be exaggerated.
Tobaceo has been accused of contributing to the excitement in the new habit any more than
one who is beginning to drink. He simply imitates
the bearded persons whom he sees with the pipe or Tobacco has been accused of contributing to the the bearded persons whom he sees with the pipe or
depopulation of the country by enfeebling the repro- cigar in their mouths. It is to him one of the signs of
ductive powers of men and inducing miscarriages in the virility to which he aspires. It is the easiest way women. The former part of the charge is founded on for him to make himself believe that he is already a its influence prevails, appeases all ardor ; but its action! but few smokers can find any traces of this feeling in general powers of smokers. Their families are as one's virility and doing like others may explain the
numerous as those of other person, and the peoples first essays in the face of the pains that attend them, who smoke most are precisely those who have the most it does not account for the irresistible attraction of the
children. The Germans smoke twice as much as the habit once formed and the readiness with which it French, and have five times as many children. The establishes itself. The customs and tastes of popula-
powsilility of tobacco promoting abortions is more tions and the fashions change and give place to others popen to diselussion, but it cannot exert any noticeable that disappear in their turn, after having inspired the
influence on the movement of population, for it con- same infatuation in us. but the habit of smoking influence on the movement of population, for it con- same infatuation in us; but the habit of smoking goes

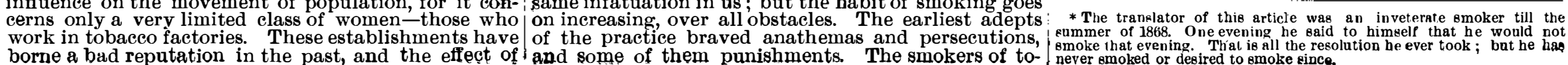
There must, therefore, be in this passion something
besides the satisfaction of a mechanical habit. "The pesidicular intoxication of caused by tobacco," says $M$. intoxicant of so recent discovery, the initiation into which is so painful, to have overtaken wine, old as the world." The charm of tobacco intoxication is not
easy to explain. It is in the soothing, says M. Fay ; it Richet; it is a state of torpor which conduces to revelry, say others. Tolstoi maintains that it is noth-
ing of this kind, but the desire to stifle the voice of conscience; and, confounding tobacco with alcohol
and opium, the Russian romancist envelops them view in the same anathema. In explanation of hise to a theory known in phygiology
as that of duality, or human dynamism. Onring his conscious life, Tolstoi says, man has frequent occasion
to recognize in himself two distirict beings : one blind and sensitive, the other enlightened and thinking.
The former eats, drinks, rests, sleeps, reproduces, and moves, like a machine wound up for a certain time.
The other, the thinking and enlightened, united with The other, the thinking and enlightened, united with
the sensitive one, does not act by itself, but only con-
trols and appraises the conduct of the former one, trols and appraises the conduct of the former one,
helping it effectively if it approves, and remaining
neutral in the contrarycase. This spiritual but powerneutral in the contrarycase. This spiritual but powercompass of the ship, of which the other being is the
helmsman. The last can follow the directions of the me is even able, when its warnings annoy him, to disarrange his compass. Weak and timorous persons
have recourse to the last expedient. They stifle their
conscience, and, in order to do so, use alcohol or Count Tolstoi's theory cannot be sustained. It has one particularly weak point in the similarity which
the author assumes between the effects of tobacco and
of alcohol. Not one of the personages whom the translator of his work consulted protested against this con-
fusion, and still it is false and deceitful. The Rusfusion, and still it is false and deceitful. The Rus-
sian's paradox may be applied, to a certain extent, to
drunkenness. We do sometimes get drunk to forget to stupefy ourselves, and it is a detestable means.
Rogues and criminals all do it; they drink of ten to give themselves heart, murderers especially; while oberved, of a erime committed with pipe or cigar in
the mouth. The author himself confesses that he dulled his conscience with tobacco for a long time. It Sometimes it reproved him for idleness, or admonished
him for a neglect, or a want of punctuality, or an excess of passion in which he had not measured his all was forgotten. If tobaceo had never committed
worse misdeeds, nobody, I believe, would have thorse misdeeds, nobody,
Other modes of voluntary it.

Other modes of voluntary intoxication have the com-
mon characteristic of cleranging the reason and the moral sense. Hashish produces hallucinations and delirium, and plunges persons into a condition like
madness. Opium puts to sleep, and procures for some madness. Opium puts to sleep, and procures for some
perwons agreeable dreams; but one becomes quickly
hat it urtcel to it, the doses have to be increased, all the functions flag, and the opium smoker falls into a condition of inanity, at times interrupted by fits of
homicidal furor. Morphinomaniacs do not suffer the same perversion of mind, but they become false, dis-
simulating, indifferent to all that is foreign to their pamulating, indifferent to all that is foreign to their
passion, extending to family feeling and even to honor Their health is injured more quickly than by opium
smoking, and their life is shortened as much. Alcohol-
ism is still worse. I have studied its effects in all their phases in another work, and will not repeat my con-
clusions now. It is sufficient to recollect that the ignoble and degrading vice attacks nations in all their vital forces; families in their honor, fortune, and pros-
perity; that it peoples hospitals, insane asylums, and prisons; and

Tobaceo can be reproached with no such mischief.
thas never led the reason astray, destroyed the will It has never led the reason astray, destroyed the will,
or perverted the sensibility of any one. The most
hardened smoker enjoys at all times the most perfect clearness of mind. Even at the moment when he is stuer the influence of nicotine he talks, reasons,
proves that horks with a freedom of thought that proves that his intelligence has not received any harm. physieal impressions and that, as Dr. Riehtent says, it
nnollifies the sensibility of the organs only to leave the psychical functions greater freedom of evolution.

There is another characteristic difference between
tobacco and other voluntary poisons. A person can break up the habit of using tobacco, while alcoholism
and morphinomania are almost incurable. At the end of my long career I cannot recollect having witnessed cannot affirm that they would have been permanent
if the subjects had been exposed to new temptations.
Morphinomaniaes are absolutely incurable unless they are interned. Smokers, on the other hand, can cor-
reet themselves when they wish to. They only need a firm will. We see persons every day who have done
this ; and since the troubles caused by tobaceo have this ; and since the troubles caused by tobacco have
been more definitely known, we see many men giving
it up of their own accord as they advance in age. The habit is so completely lost that after a few years the reformed without feeling a desire to imitate them ; and
if he is moved to light a cigar, he will not find the pleasure of the old days in it.
I might stop here; but I will not finish this article without giving my own explanation of the fascination
of tobacco. It is probably no better than the others, and I will not try to impose it on any one.
Men have at all times eagerly sought for substances
that would act on their nervous system. Th tendency 
is general, and is exclusively human. To escape real case, the earth would leave the side walls through in order to increase the picturesque effect, a certain life and the drudgery of daily occupations, to live in the effect of rain, and accumulate at the bottom. number of pockets are formed irregularly in the excan people at its will, and can embellish with its illusions, have irresistible charms to some minds. In
obedience to this dangerous seduction they involuntarily seek the dreams of opium and hashish, the intoxi
cation of ether and chloral, or the grosser drunkenness cation of ether and chloral, or the grosser drunkenness
of alcohol. The weak yield unresistingly to their inclination, and pass into the degrading excesses which and is attended with no such dangers. Its action on the nervous system is weak and wholly special. It
does not put to sleep, but it calms and mollifies the sensibility of the organs. It causes an agreeable torpor, during which thought continues lucid, and the th traction it exercises, and which causes it to be sought for by so many thinkers and students. Tobacco is to them a help in mental labor. When fatigue begins and the need of a moment's rest is felt; when the thought fails to present itself with the usual exactness,
and the mind hesitates over the shape to give it, the student, writer, or investigator stops, lights his pipe, thought appears clear and limpid through the bluish cloud in which the smoker has enveloped himself. I should make a wrong impression if I left it to be labor. It becomes so only for those who have contheted the habit of oning it, and they can divore themselves from it without losing their capacity. As have a mischievous influence on the health, and may cause serious diseases. We should not advise any one to use it, and should try to keep women and children
from doing so. In taking up this part of its programme, and in affiliating itself with teachers of all grades, the Society against the Abuse of Tobacco ha performed real service; but it has tried to gain its end no use, and would be labor lost, to try to convert adult smokers so long as they experience no inconvenience from the habit. As soon as they begin to feel some
troubles, and have reached an age when the troubles may become grave, the dangers to which they are
exposing themselves should be described to them without extenuating them, but without making the picture blacker. If dangerousaffectionsare threatened, like angina pectoris, or injuries to the tongue and lips, a decisive course must be taken, and the immedipipe insisted upon, for experience has taught that there can be no gradual leaving off.-Translated for
The Popular Science Monthly from the Revue des Deux The Poputar
Mondes.

\section{ALPINE GARDENS.*}

THE first alpine gardens were suggested by the sight of torrents which, dried up during the summer, and snow, have their steep banks covered with a multitude of charming plants during the fine season. This idea should not be lost sight of by him who undertakes such a work, and the result will be so much the better
in proportion as it more accurately reproduces the in proportion as it more accurately reproduces the leisure for it, the artist should go to seek the combinations that satisfy the eye from all points, in situ, note the form and dimensions of each rock, the relative positions of the plants that cover or surround it, and fix each of these details by an accurate sketch or by photography. The art will afterward consist in groupa perfect whole. a perfect whole. with the exigences of culture. The amateur should therefore, possess taste and a certain amount of horticultural knowledge; otherwise, it will be well for him to ask advice of a landscape gardener. The first con-
dition to be fulfilled is to give sufficient nourishment to the plants that are to embellish the rocks. In most in the soil Although some species, like the orpines (Sedum), the house leeks (Sempervivum), the saxifrages, etc., live upon the bare rock the great majority of alpine plants seek deep earth and develop roots therein several feet in length, such (Dianthus alpinus and others), the globe daisies way that horizontal fissures, such as shown by No 1 of Fig. 1, are not, as a general thing favorable to the culture of alpine plants. When superposed rocks are used, it will be well to spread a good layer of suitable
soil over each of them before placing the succeeding one, and to maintain the necessary spacing by frag In most hases it will be preforale or gran to (No. 2). vertical fissures between the rocks, but care must be taken that the upper rocks do not overhang. A plant placed at the entrance of the fissure, $\mathrm{H}$, as shown by No. 3 , will receive the sun and the rain, while placed
at $K$ (No. 4), it will soon perish for want of light and A satisfactory form for the establishment of ragged rocks is that shown by No. 5. The rain falls suceessively upon each stone and infilters into each fissure and reaches the bottom of it. For the same reason, it is well to cover the upper part of the work with earth unless, however, it is desired to produce a definit picturesque effect.

vertical fissures, which are those best adapted to alpine plants, should, as far as possible, be narrower
at the bottom than at the top. Were it otherwise the

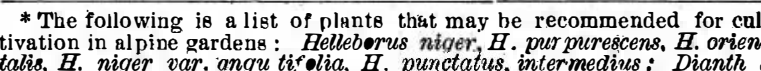

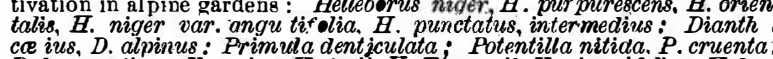

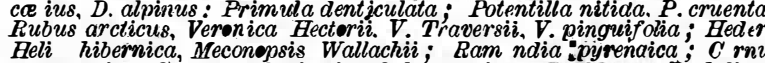

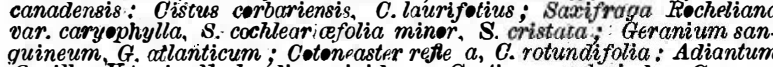

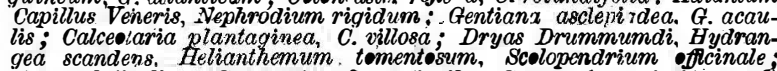

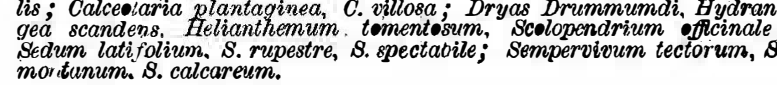

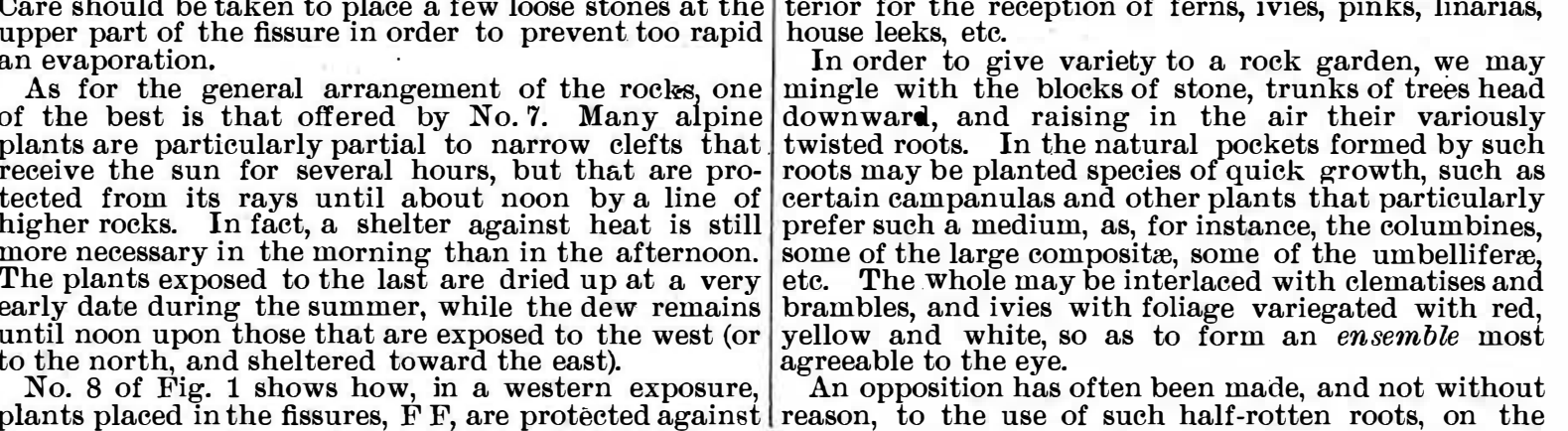
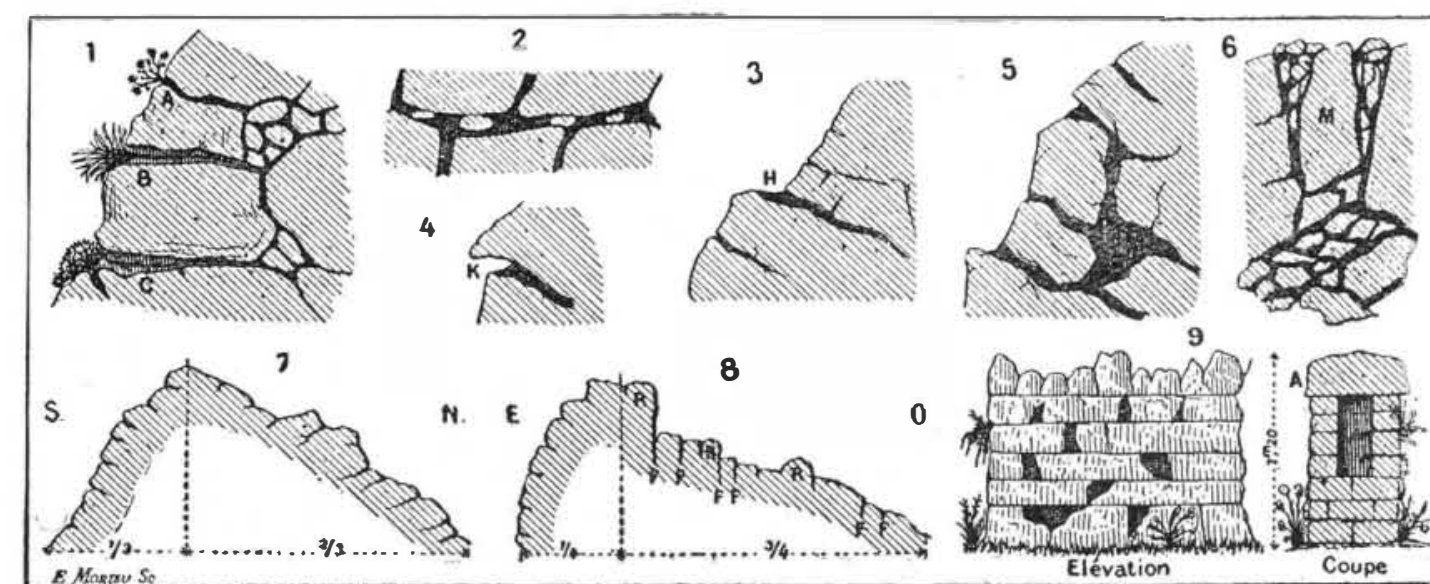

8

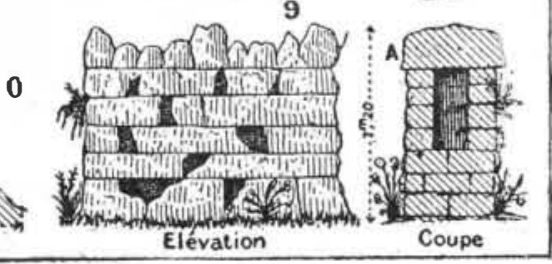

FIG. 1.-ARRANGEMENT OF ROCKWORK IN ALPINE GARDENS. he morning heat of the sun by the rocks, R R, forming ground that they decompose too rapidly, lose their e selected for the culture of alpine plants are the woodlice. This second part of the criticism is very western and northern ones, provided.that, in the latter just, and we could not too strenuously recommend In they are sheltered toward the east. fome already existing wall, or to construct one, to und picturesque manner of utilizing such a wall for the culture of alpine plants and especially of alpine the narrow sides of the rocks (No. 9).
. A width of about 24 in. is given the base of this wal and a height of $20 \mathrm{in}$. above the surface of the ground
The two sides, which must be made of rubble, hould have a space between to be filled in with carefully prepared earth. Finally, the whole is covered the stability of the structure. in which a number of alpine plants grow admirably. and replaced by earth, and it is into these interstices devote his attention to dig ging deeply into the earth that are introduced the roots of the alpine plants so that the roots may easily descend therein and find

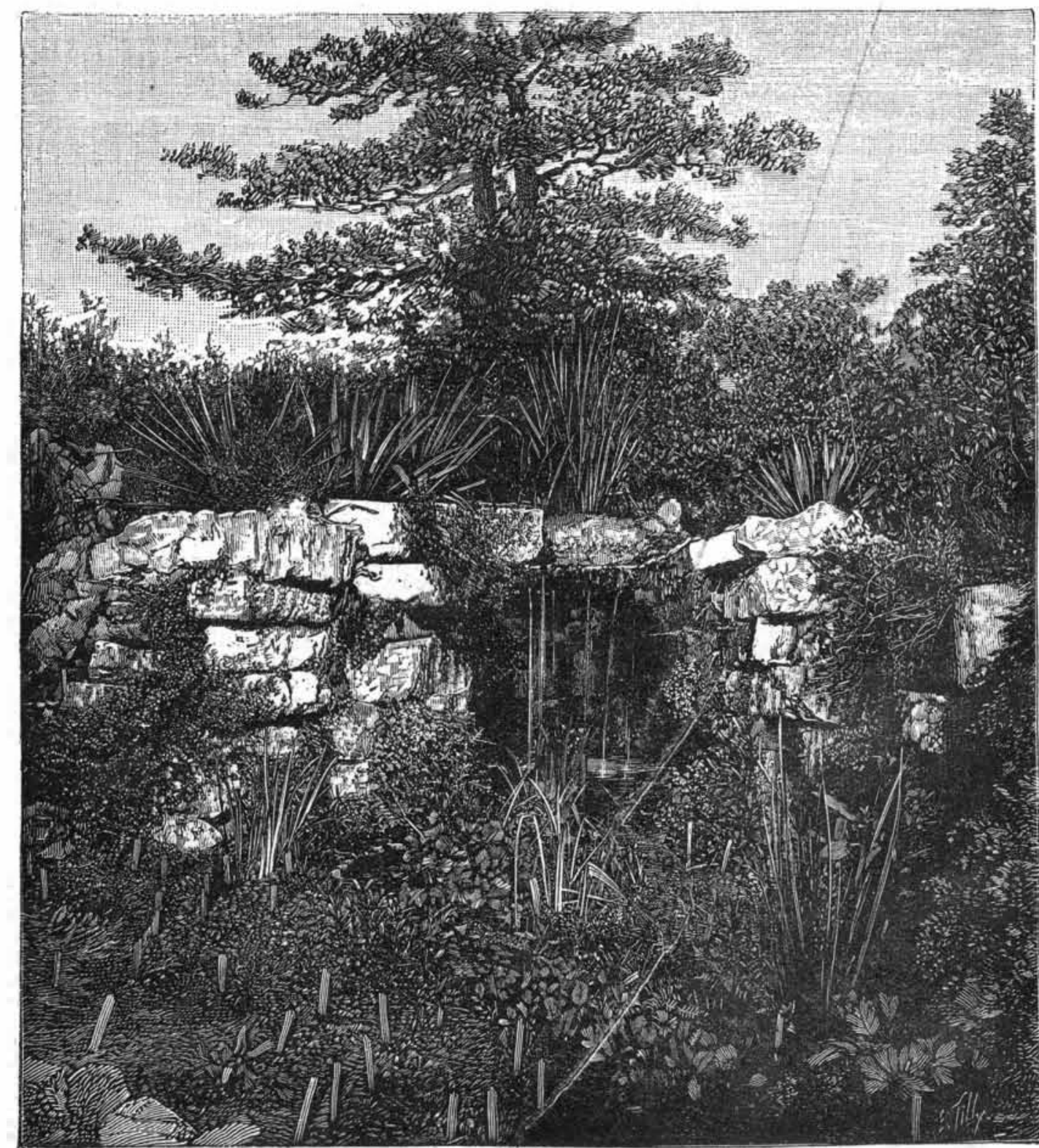

Fig. 2.-ROCK GARDEN AT KEW-CASCADE AND AQUATIC PLANTS. 